\title{
ORIGINAL
}

\section{Clinical features, ventilatory management, and outcome of ARDS caused by COVID-19 are similar to other causes of ARDS}

\author{
Carlos Ferrando 1,2* $\mathbb{D}$, Fernando Suarez-Sipmann 2,3,4, Ricard Mellado-Artigas', María Hernández ${ }^{5}$, Alfredo Gea ${ }^{6}$, \\ Egoitz Arruti ${ }^{7}$, César Aldecoa ${ }^{8}$, Graciela Martínez-Pallí ${ }^{1}$, Miguel A. Martínez-González ${ }^{9,10}$, Arthur S. Slutsky ${ }^{11,12}$ \\ and Jesús Villar2,11,13 on behalf of the COVID-19 Spanish ICU Network
}

(c) 2020 Springer-Verlag GmbH Germany, part of Springer Nature

\begin{abstract}
Purpose: The main characteristics of mechanically ventilated ARDS patients affected with COVID-19, and the adherence to lung-protective ventilation strategies are not well known. We describe characteristics and outcomes of confirmed ARDS in COVID-19 patients managed with invasive mechanical ventilation (MV).

Methods: This is a multicenter, prospective, observational study in consecutive, mechanically ventilated patients with ARDS (as defined by the Berlin criteria) affected with with COVID-19 (confirmed SARS-CoV-2 infection in nasal or pharyngeal swab specimens), admitted to a network of 36 Spanish and Andorran intensive care units (ICUs) between March 12 and June 1, 2020. We examined the clinical features, ventilatory management, and clinical outcomes of COVID-19 ARDS patients, and compared some results with other relevant studies in non-COVID-19 ARDS patients.

Results: A total of 742 patients were analysed with complete 28-day outcome data: 128 (17.1\%) with mild, 331 (44.6\%) with moderate, and 283 (38.1\%) with severe ARDS. At baseline, defined as the first day on invasive MV, median (IQR) values were: tidal volume $6.9(6.3-7.8) \mathrm{ml} / \mathrm{kg}$ predicted body weight, positive end-expiratory pressure 12 (11-14) $\mathrm{cmH}_{2} \mathrm{O}$. Values of respiratory system compliance $35(27-45) \mathrm{ml} / \mathrm{cmH}_{2} \mathrm{O}$, plateau pressure $25(22-29) \mathrm{cmH}_{2} \mathrm{O}$, and driving pressure $12(10-16) \mathrm{CmH}_{2} \mathrm{O}$ were similar to values from non-COVID-19 ARDS patients observed in other studies. Recruitment maneuvers, prone position and neuromuscular blocking agents were used in $79 \%, 76 \%$ and $72 \%$ of patients, respectively. The risk of 28-day mortality was lower in mild ARDS [hazard ratio (RR) 0.56 ( $95 \% \mathrm{Cl} 0.33-0.93)$, $p=0.026$ ] and moderate ARDS [hazard ratio (RR) 0.69 (95\% Cl 0.47-0.97), $p=0.035$ ] when compared to severe ARDS. The 28-day mortality was similar to other observational studies in non-COVID-19 ARDS patients.

Conclusions: In this large series, COVID-19 ARDS patients have features similar to other causes of ARDS, compliance with lung-protective ventilation was high, and the risk of 28-day mortality increased with the degree of ARDS severity.
\end{abstract}

Keywords: Acute respiratory distress syndrome, Coronavirus, Mechanical ventilation, Outcome

\footnotetext{
*Correspondence: cafeoranestesia@gmail.com

${ }^{1}$ Department of Anesthesiology and Critical Care, Institut D'investigació

August Pi I Sunyer, Hospital Clínic, Villarroel 170, 08025 Barcelona, Spain

Full author information is available at the end of the article
}

\section{实




\section{Introduction}

In late December 2019, the Chinese Center for Disease Control and Prevention (Chinese CDC) reported a series of cases of unknown pneumonia which was subsequently termed Coronavirus disease 2019 (COVID-19), caused by the severe acute respiratory syndrome coronavirus 2 (SARS-CoV-2) [1]. The health, social, and economic impact of this disease is unprecedented in our life-time. The COVID-19 pandemic has collapsed health care systems and led to an overwhelming pressure on Intensive Care Units (ICUs), since many patients developed profound hypoxemia and extensive pulmonary infiltrates requiring intubation and ventilatory support [2].

Recent publications from China and Italy have described the epidemiology, clinical characteristics, and prognostic factors of patients who developed acute respiratory distress syndrome (ARDS) caused by COVID19 [3-5]. A number of editorials and anecdotal points of view have suggested that COVID-19 ARDS has an atypical behavior, since a number of patients with profound hypoxemia had normal or close to normal respiratory system compliance (Crs) [6-8]. However, data confirming this assumption are scarce, and the view that severe COVID-19 causes an "atypical" ARDS has generated debate. Consequently, there is controversy as to what are the most appropriate oxygenation and ventilation strategies without increasing ventilation-induced lung injury or multi-organ damage.

It has long been known that patients with ARDS have markedly varied clinical presentations, and the Berlin definition did not include a threshold value for respiratory compliance as a diagnostic criterion for ARDS, because it did not add to predictive validity [9]. As well, it can be difficult to measure accurately in non-passive patients.

The clinical features of patients with SARS-CoV2-induced ARDS, and the ventilatory management, and patient outcomes have not been well described [4]. The main objective of this large observational study was to describe the physiologic characteristics over time, the ventilatory management, and outcomes in a large cohort of confirmed ARDS COVID-19 patients. A secondary objective was to compare respiratory parameters and outcomes of ARDS COVID-19 patients with ARDS of other causes, where possible.

\section{Methods}

\section{Study design}

This is a prospective, multicenter, observational, cohort study that enrolled patients with COVID-19 ARDS admitted into 36 hospitals from Spain and Andorra (participating centers are listed in the Supplementary file).

\section{Take-home message}

The COVID-19 pandemic has collapsed health care systems and led to a critically overwhelming pressure on Intensive Care Units (ICUs), since many patients developed profound hypoxemia and extensive pulmonary infiltrates requiring intubation and ventilatory support. COVID-19 patients with ARDS predominantly presented a typical moderate-to-severe ARDS. Ventilatory management, and 28-day outcome did not differ from other causes of ARDS.

During the pandemic, there were no specific hospitals that were designated as COVID-19 centers, and thus the distribution of patients among centers was similar to that observed pre-COVID-19. The study was approved by the referral Ethics Committee of Hospital Clínic, Barcelona, Spain (code number: HBC/2020/0399). According to Spanish legislation, this approval is valid for all participating centers. The informed consent was waived, except in three centers where the institutional review boards requested oral informed consent from patient's relatives. This study followed the "Strengthening the Reporting of Observational Studies in Epidemiology (STROBE)" statement guidelines for observational cohort studies [10].

\section{Study population and data collection}

Data from patients' electronic medical records were reviewed and collected by physicians trained in critical care, according to a previously standardized protocol. Each investigator had a personal username and password and entered data into a specifically pre-designed online data acquisition system (CoVid19.ubikare.io). Patient confidentiality was protected by assigning a de-identified patient code. All consecutive COVID-19 patients included in the dataset from March 12 to June 1,2020 were enrolled if they fulfilled the following criteria: $\geq 18$ years old, intubated and mechanically ventilated, confirmed SARS-CoV-2 infection from a respiratory tract sample using PCR-based tests, and had acute onset of ARDS, as defined by the Berlin criteria [9], which includes a new or worsening respiratory symptoms due to COVID infection, bilateral pulmonary infiltrates on chest imaging (X-ray or CT scan), absence of left atrial hypertension or no clinical signs of left heart failure, and hypoxemia, as defined by a ratio between partial pressure of oxygen in arterial blood $\left(\mathrm{PaO}_{2}\right)$ and fraction of inspired oxygen $\left(\mathrm{PaO}_{2} / \mathrm{FiO}_{2}\right) \leq 300 \mathrm{mmHg}$ on positive end-expiratory pressure (PEEP) $\geq 5 \mathrm{cmH}_{2} \mathrm{O}$, regardless of $\mathrm{FiO}_{2}$. Exclusion criteria were patients with non-confirmed SARS-CoV-2 infection according to WHO guidance [11], patients with no data at baseline, patients with no information on ventilatory parameters, or non-intubated patients. 
Recorded data included demographics [age, gender, body mass index (BMI), comorbidities], vital signs [temperature, mean arterial pressure (MAP), heart rate], laboratory parameters (blood test, coagulation, biochemical), ventilatory parameters [tidal volume (VT), inspiratory oxygen fraction $\left(\mathrm{FiO}_{2}\right)$, respiratory rate (RR), PEEP, plateau pressure (Pplat), driving pressure (DP), respiratory system compliance $(\mathrm{Crs})]$, the use of adjunctive therapies [recruitment maneuvers (RM), prone position, neuromuscular blocking agents (NMBA), extracorporeal membrane oxygenation (ECMO)], pharmacological treatments, disease chronology [time from onset of symptoms and from hospital admission to initiation of mechanical ventilation (MV), ventilator-free days (VFDs) during the first 30 days, ICU length of stay (LOS)]. Sequential Organ Failure Assessment (SOFA) and APACHE II scores, patients discharged from ICU, patients who had died or still being treated in the ICU on June 1, 2020 were also reported.

A full data set was obtained on the first day on invasive MV which was defined as baseline. We also collected the "worst" values during the period of invasive respiratory support (maximum or minimum, depending on the parameter). Site investigators collected what they considered to be the most representative data of each day from admission to ICU discharge, alive or dead. Prior to data analysis, two independent investigators and a statistician screened the database for errors against standardized ranges and contacted local investigators with any queries. Validated or corrected data were then entered into the database.

\section{Statistical analysis}

For the main objective of the study, two descriptive analyses including clinical characteristics, mechanical ventilation data, respiratory parameters, and adjunctive measures were performed. First, we describe patients stratified as mild, moderate, and severe ARDS based on the Berlin criteria. Second, we describe patients stratified as having normal $\mathrm{Crs}\left(\geq 50 \mathrm{ml} / \mathrm{cmH}_{2} \mathrm{O}\right)$ or low $\mathrm{Crs}$ $\left(<50 \mathrm{ml} / \mathrm{cmH}_{2} \mathrm{O}\right)$ according to baseline values [12]. Patients were considered as having low $\mathrm{Crs}$ if $<50 \mathrm{ml} /$ $\mathrm{cmH}_{2} \mathrm{O}$ on day 1 of invasive MV. Descriptive variables are expressed as percentage, mean and standard deviation (SD), or median and interquartile range (IQR), as appropriate. Then, we compared variables across groups using Student's $t$ test or Mann-Whitney test and one-way ANOVA or Kruskal-Wallis test for numerical variables, and Chi-squared test or Fisher exact test for categorical variables. Second, to assess the relationship among ARDS severity and discontinuation from mechanical ventilation, ICU discharge and mortality at day 28 time to event curves were plotted using the Kaplan-Meier method and analyzed with log-rank test and univariable Cox regression analysis. The same analysis was performed for Crs. Time to discontinuation from mechanical ventilation/ mortality/ICU discharge was described using KaplanMeier plots across categories of ARDS severity, Crs, plateau pressure and driving pressure. For the Kaplan-Meier analyses, patients with the complementary outcome were right-censored at the longest recorded length of stay. Additionally, to test differences between groups, we used log-rank test and univariable Cox regression model due to the absence of imbalances between groups at baseline (or multivariable, adjusted for ARDS, in the case of plateau pressure and driving pressure). As a sensitivity analysis, we reported results using competing-risks approach. Results were consistent across methods [12]. We compared our results for Crs, Pplat, and driving pressure to five studies in the literature [13-17] using one sample Student's $t$ test. For the largest study (LUNG SAFE), we estimated median Crs from Supplemental Figure e2, since it was not explicitly reporter in the study. When mean values of the whole cohort were not reported, we calculated it from the mean values of the study groups.

As this was an observational study with no harm or benefit to patients in the study, we aimed to recruit as many patients as possible, with no pre-defined sample size. All time to events were defined from day 1 of invasive MV. Missing data were not imputed. Analyses were performed in a complete case analysis basis. All tests were two-sided, and a $\mathrm{p}$-value $<0.05$ was considered statistically significant. We have applied the BenjaminiHochberg corrections procedure, and have marked with an asterisk the $p$ values that were $<0.05$ after the correction. All analyses were performed with STATA version 16.

\section{Results}

\section{Characteristics}

Over a period of 81 days (between 12 March and June 1, 2020), 742 mechanically ventilated patients admitted to 36 ICUs were included in the study and followed for at least 28 days (Fig. 1). The distribution of included patients among the different participating hospitals is shown in Table S1. The enrollment and follow-up of patients are still ongoing, and as of June 29 2020, 100 (13\%) patients were still in the ICU. Demographics, APACHE II and SOFA scores, vital signs and laboratory findings at baseline are shown in Table 1 and Table S2. The percent of patients with severe, moderate and mild ARDS was $38.1 \%, 44.6 \%$ and $17.2 \%$, respectively (Table 1 ); the percentage of severe ARDS patients was higher than a number of other large observational studies in nonCOVID-19 ARDS patients. 


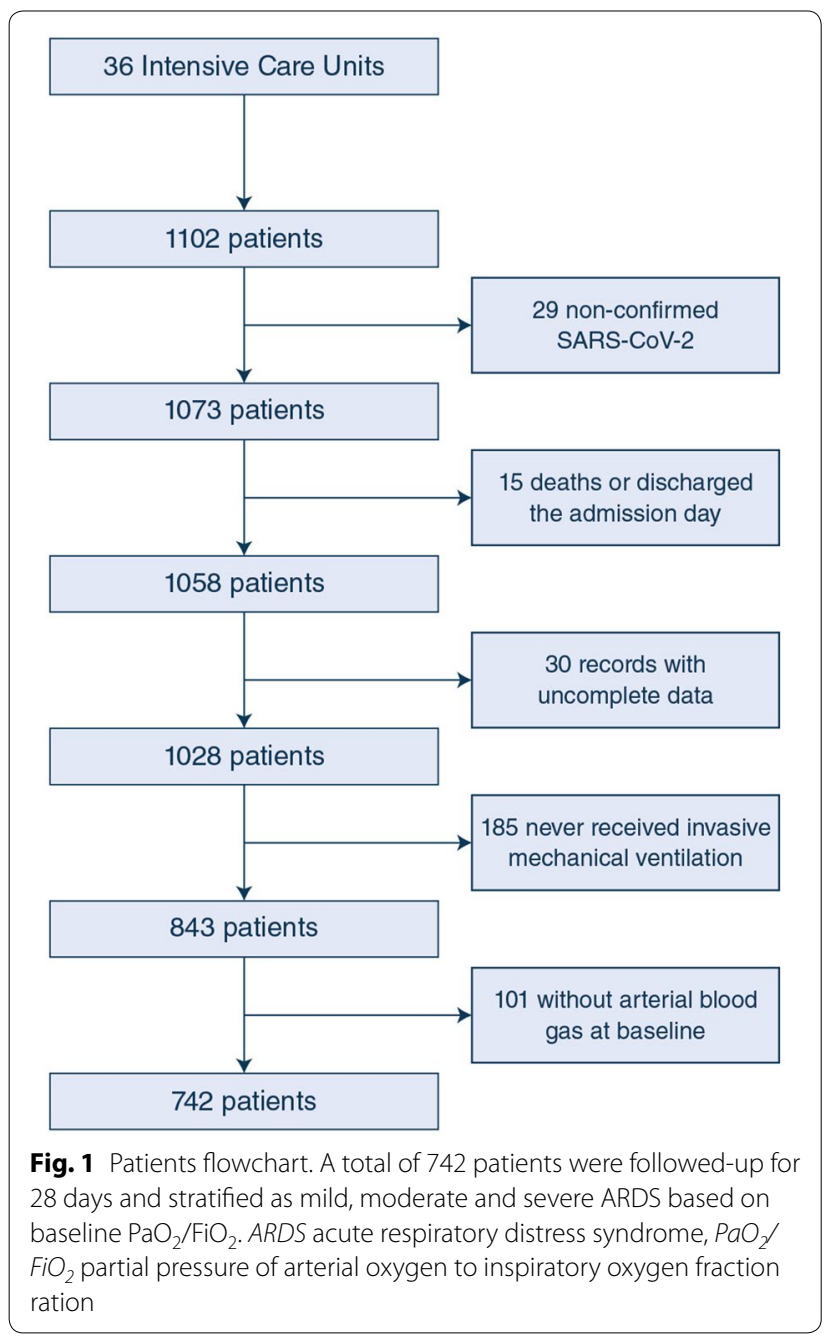

The percent of patients with severe ARDS decreased markedly from Day 1 to Day 2 and remained at this lower level from day 2 onwards (Fig. 2). This was paralleled by an increase in the percentage of patients with mild ARDS. From the 296 patients (40.8\%) with compliance data, 78\% (231) were classified as having low Crs (Tables S2, S3 and Figure S1). From these 296 patients, 35.7\% were classified as severe, $44.4 \%$ as moderate and $18.9 \%$ as mild.

\section{Mechanical ventilation and respiratory parameters}

Median time from the onset of symptoms to initiation of invasive MV was 12 (IQR: 9-16) days, and from hospital admission to initiation of invasive MV was 5 (IQR: 2-8) days. The median VT at baseline was 6.9 (IQR: 6.3-7.8) $\mathrm{ml} / \mathrm{kg}$ predicted body weight (PBW); in $23 \%$ of patients the VT never exceeded $6 \mathrm{ml} / \mathrm{kg}$ PBW. The median highest VT, including during the weaning process with assist modes, was 8.4 (IQR: 7.3-9.5) $\mathrm{ml} / \mathrm{kg}$ PBW. The median PEEP at baseline was 12 (IQR: $11-14$ ) $\mathrm{cmH}_{2} \mathrm{O}$, similar to the highest collected values of 14 (IQR:12-15) $\mathrm{cmH}_{2} \mathrm{O}$ (Table 1). Mean VT and PEEP during MV are shown in Figures S2 and S3. The ventilation strategy (VT and PEEP) did not vary with the degree of lung severity or with Crs (Table 2 and S3). The median $\mathrm{PaO}_{2} / \mathrm{FiO}_{2}$ at baseline was 120 (IQR: $83-177) \mathrm{mmHg}$. The lowest values reported during the patient's evolution was 84 (IQR: 65-114) $\mathrm{mmHg}$.

At baseline, median values for Crs, Pplat and driving pressures were 35 (IQR: $27-45$ ) $\mathrm{ml} / \mathrm{cmH}_{2} \mathrm{O}, 25$ (IQR: 22-29) $\mathrm{cmH}_{2} \mathrm{O}$, and 12 (IQR: $\left.10-16\right) \mathrm{cmH}_{2} \mathrm{O}$, respectively (Table 2). These values were not statistically different from values obtained from a number of large relatively recent observational and randomized studies of ARDS patients (Table S4).

The worst values during the MV period were 29 (IQR: 22-37) $\mathrm{ml} / \mathrm{cmH}_{2} \mathrm{O}, 28$ (IQR: 23-31) $\mathrm{cmH}_{2} \mathrm{O}$, and 15 (IQR: $12-19) \mathrm{cmH}_{2} \mathrm{O}$, respectively. Figures $\mathrm{S} 4$ and $\mathrm{S} 5$ show mean values during controlled MV. There were no differences in oxygenation $\left(\mathrm{PaO}_{2} / \mathrm{FiO}_{2}\right)$ between patients with normal or low Crs (Table S3). Although the distribution of patients with normal or low Crs showed significant differences in driving pressure, both at baseline [8 (IQR: 6-9) vs 14 (IQR: $12-17) \mathrm{cmH}_{2} \mathrm{O}, p<0.001$ ] and at maximum values [10 (IQR: $8-13$ ) vs 16 (IQR: $13-20$ ) $\left.\mathrm{cmH}_{2} \mathrm{O}, p<0.001\right]$, these differences were not associated with ARDS severity (Table 2 and Figure S5).

\section{Adjunctive measures}

Continuous NMBA were used in $72 \%$ of patients, prone position in 76\%, and RM in 79\%. Degree of ARDS severity was associated with significant differences in the use of prone position $(p<0.001)$ and NMBA $(p=0.01)$, but not RM (Table 2, Figure S6). No differences were observed in patients with normal vs low Crs (Table S3 and Figure S7). The pharmacological treatments received by the patients is shown in Table S5.

\section{Clinical outcomes}

Mean VFDs (to day 30) was 14 [IQR: 3-20] days. As of June 29, 2020, 401 (54\%) patients were discharged from the ICU with an ICU LOS of 19 [IQR: 11-37] days. Allcause 28 -day mortality was $32 \%$ (241 patients) distributed as $39 \%$ in severe, $29 \%$ in moderate and $24 \%$ in mild ARDS (Table 2). These mortality values were similar to those from four observational studies from the past 10 years (Table S6). The probability of discontinuation of MV was not significantly affected by ARDS severity (Fig. 3). The probability of ICU discharge was higher in mild [hazard ratio (RR) 1.49 (95\% CI 1.08-2.04), $p=0.014$ ], but not in moderate when compared to severe ARDS (Table 2 and Fig. 3). The risk of 28-day mortality was lower in mild ARDS [hazard ratio (RR) 0.56 (95\% CI 0.33-0.93), 
Table 1 Patient characteristics according to ARDS severity

\begin{tabular}{|c|c|c|c|c|c|}
\hline & $\begin{array}{l}\text { All } \\
(n=742)\end{array}$ & $\begin{array}{l}\text { Severe ARDS } \\
(n=283)\end{array}$ & $\begin{array}{l}\text { Moderate ARDS } \\
(n=331)\end{array}$ & $\begin{array}{l}\text { Mild ARDS } \\
(n=128)\end{array}$ & $p$ value \\
\hline \multicolumn{6}{|c|}{ Patients demographics and comorbidities at baseline } \\
\hline Age $(n)$ & $64[56-71](737)$ & $64[56-71](280)$ & $64[56-71](329)$ & $64[55-71](128)$ & 0.859 \\
\hline Gender, male & $504 / 740(68.1 \%)$ & $185 / 281(65.8 \%)$ & 238/331 (71.9\%) & $81 / 128(63.3 \%)$ & 0.118 \\
\hline Body mass index, $\mathrm{kg} / \mathrm{m}^{2}(n)$ & $29[26-33](480)$ & $29[26-34](169)$ & $28[26-32](223)$ & $29[26-31](88)$ & 0.035 \\
\hline Arterial hypertension & $364 / 742(49.1 \%)$ & $143 / 283(50.5 \%)$ & $161 / 331(48.6 \%)$ & $60 / 128(46.9 \%)$ & 0.779 \\
\hline Diabetes mellitus & $180 / 742(24.3 \%)$ & $76 / 283(26.9 \%)$ & $77 / 331(23.3 \%)$ & $27 / 128(21.1 \%)$ & 0.397 \\
\hline Chronic cardiac failure & $13 / 742(1.8 \%)$ & $3 / 283(1.1 \%)$ & $7 / 331(2.1 \%)$ & $3 / 128(2.3 \%)$ & 0.459 \\
\hline Chronic renal failure & $36 / 742(4.9 \%)$ & $9 / 283(3.2 \%)$ & $19 / 331(5.7 \%)$ & $8 / 128(6.2 \%)$ & 0.219 \\
\hline Asthma & 19/742 (2.6\%) & $13 / 283(4.6 \%)$ & 6/331 (1.8\%) & $0 / 128(0 \%)$ & 0.009 \\
\hline COPD & $35 / 742(4.7 \%)$ & $15 / 283(5.3 \%)$ & 18/331 (5.4\%) & $2 / 128(1.6 \%)$ & 0.167 \\
\hline Obesity & $262 / 681(38.5 \%)$ & $112 / 262(42.7 \%)$ & $111 / 302(36.8 \%)$ & 39/117 (33.3\%) & 0.161 \\
\hline Dyslipidemia & $131 / 742(17.7 \%)$ & $57 / 283(20.1 \%)$ & $52 / 331(15.7 \%)$ & $22 / 128(17.2 \%)$ & 0.351 \\
\hline \multicolumn{6}{|l|}{ Scores } \\
\hline APACHE $\|(n)$ & $13[10-18](513)$ & 14 [10-18] (203) & $13[9-17](230)$ & $12[8-19](80)$ & 0.110 \\
\hline SOFA $(n)$ & $6[4-8](393)$ & $7[4-9](131)$ & $6[4-7](193)$ & $6[4-8](69)$ & 0.023 \\
\hline SOFA maximum (n) & $9[7-12](619)$ & $9[7-12](241)$ & $9[7-11](275)$ & $8[7-11](103)$ & 0.158 \\
\hline \multicolumn{6}{|l|}{ Vital signs } \\
\hline Temperature, ${ }^{\circ} \mathrm{C}$ & $36.6[36-37.5](708)$ & $36.8[36-37.5](269)$ & $36.5[36-37.5](316)$ & $36.6[36.0-37.1](123)$ & 0.083 \\
\hline Temperature $\max ,{ }^{\circ} \mathrm{C}$ & $38[37.4-38.7](740)$ & $38[37.5-38.8](283)$ & $38.0[37.4-38.7](330)$ & $38.1[37.4-38.9](127)$ & 0.337 \\
\hline Mean blood pressure, $\mathrm{mmHg}$ & 82 [73-93] (718) & 83 [73-95] (270) & 82 [75-91] (324) & 80 [73-90] (124) & 0.281 \\
\hline Mean blood pressure min, $\mathrm{mmHg}$ & 67 [61-74] (739) & 67 [61-73] (280) & 68 [60-75] (331) & $67[61-74](128)$ & 0.974 \\
\hline Heart rate, bpm & $80[68-96](722)$ & $86[70-100](275)$ & 80 [68-95] (322) & 78 [63-90] (125) & $<0.001^{*}$ \\
\hline Heart rate maximum, bpm & $110[95-120](740)$ & 110 [99-123] (281) & 108 [92-120] (331) & 110 [94-120] (128) & 0.025 \\
\hline \multicolumn{6}{|l|}{ Laboratory findings } \\
\hline Ferritin, ng/mL (n) & 1401 [741-2315] (271) & 1405 [767-2400] (93) & 1330 [677-1999] (125) & 1452 [793-2993] (53) & 0.574 \\
\hline Ferritin maximum, ng/mL $(n)$ & 1674 [881-2919] (578) & 1738 [918-2771] (216) & 1726 [852-3095] (259) & 1519 [780-3097] (103) & 0.910 \\
\hline D-Dimer, ng/mL (n) & 1200 [720-2620] (498) & 1200 [780-2550] (185) & 1186 [720-2487] (224) & 1219 [600-3030] (89) & 0.679 \\
\hline D-Dimer maximum, ng/mL (n) & $5455[2975-8005](700)$ & 5879 [3444-7986] (264) & $5413[2882-8085](312)$ & $4750[2439-7486](124)$ & 0.129 \\
\hline $\mathrm{CRP}, \mathrm{mg} / \mathrm{dL}(n)$ & $29[13-140](637)$ & $45[15-186](239)$ & $25[11-114](287)$ & $27[10-88](111)$ & $<0.001$ \\
\hline CRP maximum, mg/dL $(n)$ & $45[22-252](721)$ & $139[26-276](269)$ & $39[20-227](325)$ & 31 [17-203] (127) & $<0.001^{*}$ \\
\hline 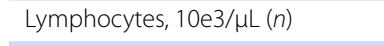 & $0.6[0.4-0.9](694)$ & $0.6[0.43-1](262)$ & $0.6[0.4-0.9](313)$ & $0.6[0.33-0.81](119)$ & 0.109 \\
\hline Lymphocytes min, 10e3/uL (n) & $0.37[0.2-0.51](725)$ & $0.38[0.22-0.53](273)$ & $0.36[0.2-0.5](325)$ & $0.32[0.2-0.51](127)$ & 0.746 \\
\hline $\mathrm{IL}-6, \mathrm{pg} / \mathrm{mL}(n)$ & 98 [29-270] (157) & $97[36-198](70)$ & $97[28-448](59)$ & $148[45-414](28)$ & 0.334 \\
\hline IL-6 max, pg/mL (n) & $224[49-986](310)$ & 313 [63-1000] (129) & 180 [49-1000] (131) & $154[40-651](50)$ & 0.406 \\
\hline Leukocytes, $10^{3} / \mu \mathrm{L}(n)$ & $9.4[6.5-13](643)$ & $9.2[6.1-13.3](256)$ & 9.7 [6.8-13.8] (284) & $8.7[6.4-11.8](103)$ & 0.160 \\
\hline Leukocytes $\max , 10^{3} / \mu \mathrm{L}(n)$ & $14.2[9.7-20.9](725)$ & 15.3 [10.6-23] (275) & $14[8.7-20.4](324)$ & $13.5[9.2-17.7](126)$ & 0.015 \\
\hline Procalcitonin, ng/mL (n) & $0.24[0.11-0.61](442)$ & $0.24[0.13-0.75](166)$ & $0.23[0.11-0.5](202)$ & $0.26[0.13-0.96](74)$ & 0.254 \\
\hline Procalcitonin max, ng/mL (n) & $0.71[0.27-3.59](645)$ & $0.85[0.3-3.84](238)$ & $0.66[0.28-3.61](290)$ & $0.70[0.23-2.9](117)$ & 0.169 \\
\hline Platelets, $1000 / \mathrm{mm}^{3}(n)$ & 234 [178-314] (712) & 237 [179-310] (270) & 235 [182-316] (320) & $220[165-301](122)$ & 0.453 \\
\hline Platelets max, $1000 / \mathrm{mm}^{3}(n)$ & $381[284-476](727)$ & $386[288-481](275)$ & 376 [290-482] (325) & 385 [273-463] (127) & 0.610 \\
\hline Bilirubin, $\mathrm{mg} / \mathrm{dL}(n)$ & $0.67[0.44-1](629)$ & $0.62[0.47-0.9](229)$ & $0.64[0.42-1](292)$ & $0.71[0.41-1.03](108)$ & 0.274 \\
\hline Bilirubin max, mg/dL (n) & $1.36[0.8-2.9](698)$ & $1.35[0.8-2.7](261)$ & $1.3[0.8-2.8](315)$ & $1.47[0.8-3.5](122)$ & 0.685 \\
\hline Troponin, ng/mL (n) & $13[4.1-39.4](335)$ & $13[0.9-39.4](114)$ & $12.8[4.1-28.5](164)$ & 18 [7-65] (57) & 0.097 \\
\hline Troponin max, ng/mL (n) & $26.3[5.9-117](568)$ & $29.6[0.9-111](202)$ & $24[6-139.9](261)$ & 27 [11.9-103] (105) & 0.246 \\
\hline
\end{tabular}

Parameters are shown at baseline (the first day on MV) and during the period of invasive respiratory support (maximum or minimum, depending on the parameter). Categorical variables are expressed as numbers (\%), and continuous variables as median (IQR)

$A R D S$ acute respiratory distress syndrome, COPD chronic obstructive pulmonary disease, SOFA sequential organ failure assessment, $R C P C$-reactive protein, IL interleukin, min minimum, max maximum

* $<0.05$ after Benjamini-Hochberg penalization 


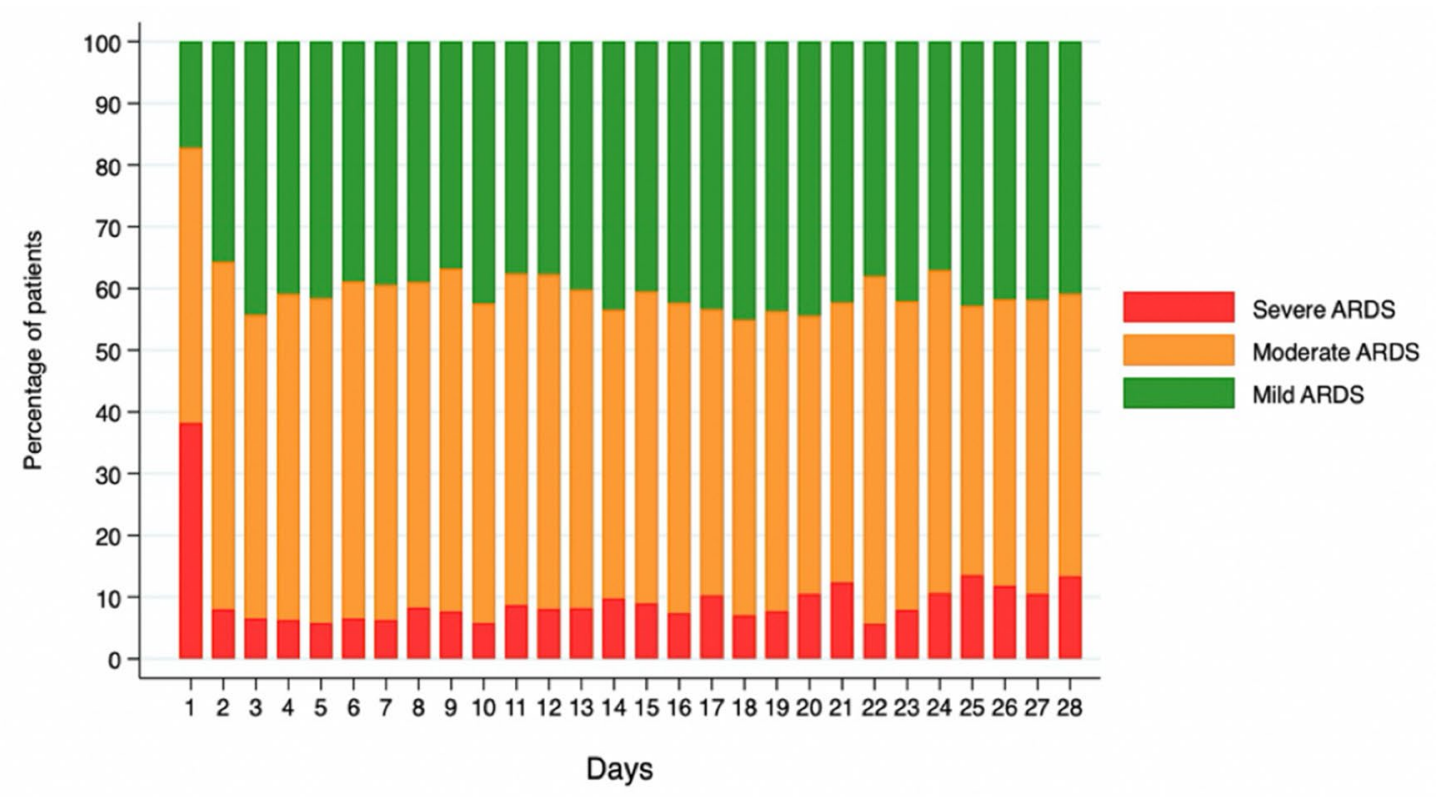

\begin{tabular}{|c|c|c|c|c|c|c|c|c|c|c|c|c|c|c|}
\hline Day & 1 & 2 & 3 & 4 & 5 & 6 & 7 & 8 & 9 & 10 & 11 & 12 & 13 & 14 \\
\hline $\mathbf{N}$ & 742 & 613 & 629 & 593 & 576 & 537 & 514 & 484 & 445 & 419 & 393 & 360 & 355 & 308 \\
\hline Day & 15 & 16 & 17 & 18 & 19 & 20 & 21 & 22 & 23 & 24 & 25 & 26 & 27 & 28 \\
\hline $\mathbf{N}$ & 281 & 257 & 244 & 215 & 208 & 191 & 170 & 142 & 152 & 132 & 126 & 110 & 105 & 105 \\
\hline
\end{tabular}

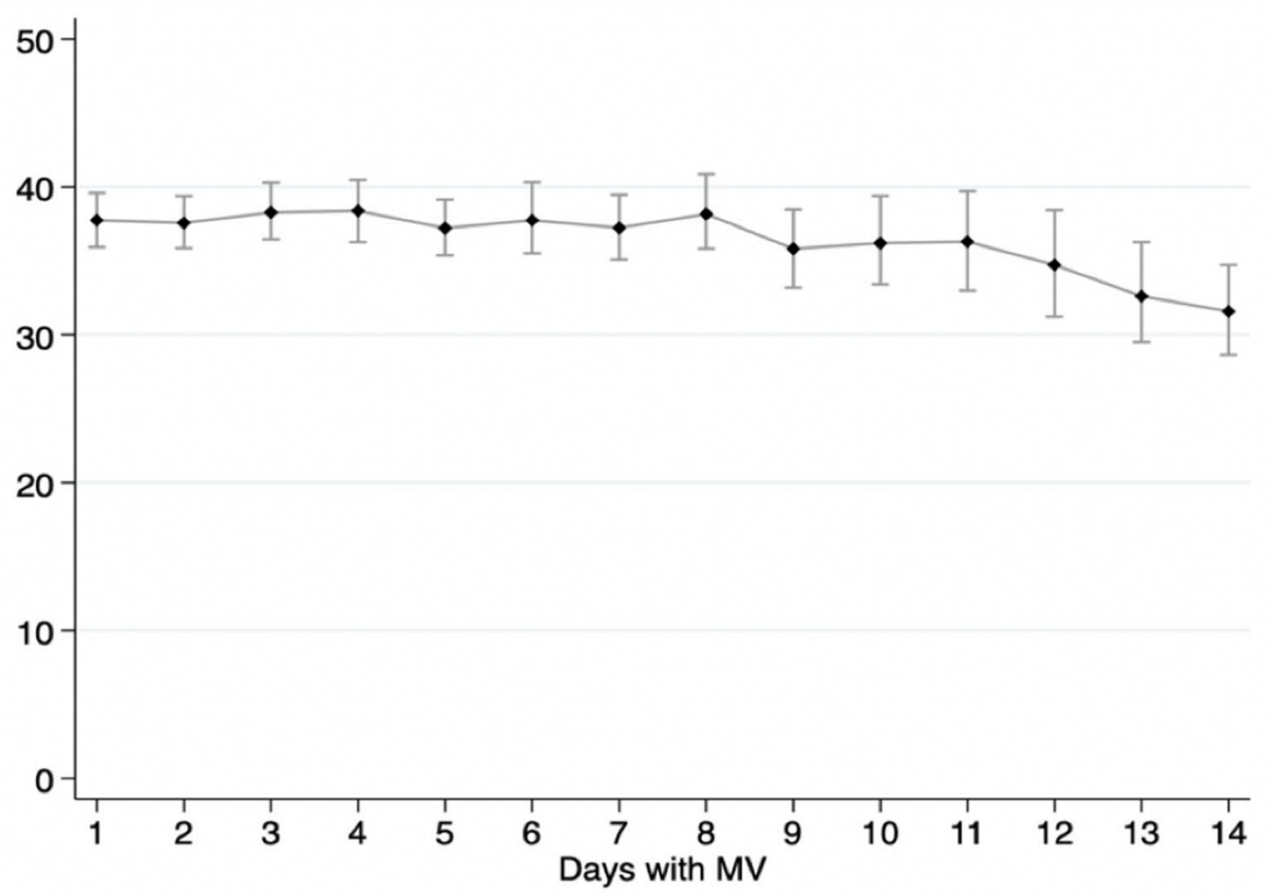

\begin{tabular}{|c|c|c|c|c|c|c|c|c|c|c|c|c|c|c|}
\hline Day & $\mathbf{1}$ & $\mathbf{2}$ & $\mathbf{3}$ & $\mathbf{4}$ & $\mathbf{5}$ & $\mathbf{6}$ & $\mathbf{7}$ & $\mathbf{8}$ & $\mathbf{9}$ & $\mathbf{1 0}$ & $\mathbf{1 1}$ & $\mathbf{1 2}$ & $\mathbf{1 3}$ & $\mathbf{1 4}$ \\
\hline $\mathbf{N}$ & 296 & 275 & 267 & 229 & 209 & 188 & 171 & 145 & 114 & 103 & 95 & 77 & 73 & 61 \\
\hline
\end{tabular}

Fig. 2 Top panel: daily distribution of patients under invasive mechanical ventilation by ARDS severity (mild, moderate, and severe) from day 1 to 28. Mild: $\mathrm{PaO}_{2} / \mathrm{FiO}_{2}<100 \mathrm{mmHg}$, moderate: $\mathrm{PaO}_{2} / \mathrm{FiO}_{2} 100-200 \mathrm{mmHg}$, severe: $\mathrm{PaO}_{2} / \mathrm{FiO}_{2}>201$ and < $300 \mathrm{mmHg}$. Bottom panel: Daily mean ( $95 \%$ confidence interval) of respiratory system compliance in $\mathrm{cmH}_{2} \mathrm{O}$. Only patients under controlled mechanical ventilation are included. ARDS acute respiratory distress syndrome, $\mathrm{PaO}_{2} / \mathrm{FiO}_{2}$ partial pressure of arterial oxygen to inspiratory oxygen fraction ration, $\mathrm{MV}$ mechanical ventilation 
Table 2 Ventilation and outcomes according to ARDS severity

\begin{tabular}{|c|c|c|c|c|c|}
\hline & $\begin{array}{l}\text { All } \\
(n=742)\end{array}$ & $\begin{array}{l}\text { Severe ARDS } \\
(n=283)\end{array}$ & $\begin{array}{l}\text { Moderate ARDS } \\
(n=331)\end{array}$ & $\begin{array}{l}\text { Mild ARDS } \\
(n=128)\end{array}$ & $p$ value \\
\hline \multicolumn{6}{|l|}{ Modes of ventilation } \\
\hline Mechanical ventilation on ICU admission (n) & $479(64.6 \%)(742)$ & $188(66.4 \%)$ & $213(64.4 \%)$ & $78(60.9 \%)$ & 0.56 \\
\hline $\begin{array}{l}\text { Days from symptoms onset to mechanical ventila- } \\
\text { tion ( } \mathrm{n})\end{array}$ & $12[9-16](734)$ & $12[9-16]$ & $12[9-17]$ & $11[8-14]$ & 0.26 \\
\hline $\begin{array}{l}\text { Days from hospital admission to mechanical ventila- } \\
\text { tion (n) }\end{array}$ & $5[2-8](742)$ & $5[2-9]$ & $4[2-8]$ & $4.5[2-7]$ & 0.51 \\
\hline \multicolumn{6}{|l|}{ Ventilatory parameters } \\
\hline Tidal volume, $\mathrm{ml}(\mathrm{n})$ & $6.9[6.3-7.8](723)$ & $6.9[6.3-7.8]$ & $7[6.3-7.7]$ & $6.9[6.3-7.9]$ & 0.919 \\
\hline Tidal volume max, ml (n) & $8.4[7.3-9.5](723)$ & $8.4[7.3-9.4]$ & $8.4[7.5-9.7]$ & $8.3[7.2-9.3]$ & 0.481 \\
\hline Tidal volume $\leq 6 \mathrm{ml} / \mathrm{kg}$, PBW (n) & $173(23 \%)(742)$ & $67(23 \%)$ & $76(23 \%)$ & $30(23 \%)$ & 0.973 \\
\hline $\mathrm{PEEP}, \mathrm{cmH}_{2} \mathrm{O}(\mathrm{n})$ & $12[11-14](716)$ & $12[10-14]$ & $12[11-14]$ & $12[12-14]$ & 0.579 \\
\hline PEEP $\max , \mathrm{cmH}_{2} \mathrm{O}(\mathrm{n})$ & $14[12-15](716)$ & 14 [12-15] & 14 [12-15] & 13 [12-15] & 0.034 \\
\hline PEEP $>12 \mathrm{cmH}_{2} \mathrm{O}(\mathrm{n})$ & $46(6.4 \%)(716)$ & $14(5 \%)$ & $25(7.9 \%)$ & $7(5.7 \%)$ & 0.348 \\
\hline Inspiratory oxygen fraction, \% (n) & $80[60-100](728)$ & $100[80-100]$ & $75[60-100]$ & $60[50-80]$ & $<0.001^{*}$ \\
\hline Mean $\mathrm{FiO}_{2}, \%{ }^{\#(n)}$ & $61[53-70](741)$ & $65[57-75]$ & $60[53-69]$ & $53[47-61]$ & $<0.001^{*}$ \\
\hline Respiratory rate, bpm (n) & $24[20-30](715)$ & 25 [20-33] & $24[20-28]$ & 23 [18-26] & $<0.001^{*}$ \\
\hline Respiratory rate max, bpm (n) & 30 [25-35] (734) & $30[27-36]$ & $30[25-35]$ & $30[25-35]$ & $<0.001^{*}$ \\
\hline Plateau pressure, $\mathrm{CmH}_{2} \mathrm{O}(\mathrm{n})$ & 25 [22-29] (215) & 25 [20-29] & 26 [22-29] & 24 [22-26] & 0.022 \\
\hline Plateau pressure $\max , \mathrm{cmH}_{2} \mathrm{O}(\mathrm{n})$ & $28[23-31](410)$ & $28[24-30]$ & $28[23-32]$ & 26 [22-29] & 0.011 \\
\hline Driving pressure, $\mathrm{CmH}_{2} \mathrm{O}(\mathrm{n})$ & $12[10-16](260)$ & 13 [9-16] & $12[10-16]$ & $12[11-14]$ & 0.473 \\
\hline Driving pressure $\max , \mathrm{cmH}_{2} \mathrm{O}(\mathrm{n})$ & 15 [12-19] (386) & 15 [12-20] & 15 [12-20] & $14[11-17]$ & 0.064 \\
\hline Respiratory system compliance, $\mathrm{ml} / \mathrm{cmH}_{2} \mathrm{O}(\mathrm{n})$ & $35[27-45](296)$ & $32[25-48]$ & $35[27-45]$ & 35 [30-49] & 0.461 \\
\hline Respiratory system compliance $\mathrm{min}, \mathrm{ml} / \mathrm{cmH}_{2} \mathrm{O}(\mathrm{n}$ ) & $29[22-37](501)$ & $27[20-35]$ & $30[22-37]$ & $32[23-40]$ & 0.052 \\
\hline Ventilatory ratio (n) & $2[1.49-2.63](610)$ & $2.09[1.53-2.71]$ & $2[1.52-2.59]$ & $1.84[1.42-2.59]$ & 0.136 \\
\hline Ventilatory ratio max (n) & $2.83[2.23-3.69](665)$ & $2.92[2.3-3.7]$ & $2.79[2.19-3.73]$ & $2.59[2.03-3.38]$ & 0.015 \\
\hline \multicolumn{6}{|l|}{ Arterial blood gases } \\
\hline $\mathrm{PaO}_{2} / \mathrm{FiO}_{2(\mathrm{n})}$ & $120[83-177](742)$ & 74 [62-88] & 142 [118-166] & 260 [222-293] & $<0.001^{*}$ \\
\hline $\mathrm{PaO}_{2} / \mathrm{FiO}_{2} \min (\mathrm{n})$ & $84[65-114](742)$ & 66 [57-80] & $104[76-125]$ & $118[85-160]$ & $<0.001^{*}$ \\
\hline $\mathrm{PaCO}_{2}, \mathrm{mmHg}(\mathrm{n})$ & $45[37-55] /(737)$ & $43[36-52] / 281$ & $46[38-56] / 329$ & $45[37-53] / 127$ & 0.026 \\
\hline $\mathrm{PaCO}_{2} \max , \mathrm{mmHg}(\mathrm{n})$ & $62[53-75](742)$ & $64[53-76]$ & $62[53-75]$ & $58[48-72]$ & 0.007 \\
\hline \multicolumn{6}{|l|}{ Adjunctive therapies } \\
\hline Recruitment maneuvers & 479/602 (79\%) & 190/237 (80\%) & $210 / 264(79 \%)$ & 79/101 (78\%) & 0.910 \\
\hline Prone & $564 / 735(76 \%)$ & 238/282 (84.4\%) & 246/327 (75\%) & $80 / 126(63 \%)$ & $<0.001^{*}$ \\
\hline Neuromuscular blockers & $536 / 742(72 \%)$ & $220 / 283(77.7 \%)$ & 234/331 (70\%) & $82 / 128(64 \%)$ & 0.011 \\
\hline ECMO & $21 / 738(2.8 \%)$ & 11/283 (3.9\%) & 9/329 (2.7\%) & $1 / 126(0.8 \%)$ & 0.232 \\
\hline \multicolumn{6}{|l|}{ Outcomes } \\
\hline Ventilation-free days & $4[0-18]$ & $0[0-16]$ & $6[0-18]$ & $8[0-21]$ & 0.069 \\
\hline Discharged from ICU & $401 / 742(54 \%)$ & 136/283 (48\%) & 185/331 (55\%) & $80 / 128(62 \%)$ & 0.017 \\
\hline Length of time on the ventilator & $14[7-24]$ & $14[8-24]$ & $14[7-24]$ & $13[7-24]$ & 0.582 \\
\hline Still in ICU & $100(13 \%)$ & $36(12 \%)$ & $47(14 \%)$ & $17(13 \%)$ & 0.880 \\
\hline Still under invasive MV & $72(9.7 \%)$ & $26(9.1 \%)$ & $34(10 \%)$ & $12(9.3 \%)$ & 1.000 \\
\hline 28-day mortality & $241(32 \%)$ & $111(39 \%)$ & $99(29 \%)$ & $31(24 \%)$ & 0.005 \\
\hline ICU length of stay & 19 [11-37] & 19 [12-35] & $19[11-39]$ & $19[11-36]$ & 0.894 \\
\hline ICU length of stay of discharge patients & $17[11-28]$ & 17 [12-28.5] & $17[11-30]$ & $17.5[10-27]$ & 0.940 \\
\hline ICU length of stay of deceased patients & 17 [10-25] & $17[11-27]$ & $17[9-26]$ & $17[10-21]$ & 0.803 \\
\hline
\end{tabular}

Parameters are shown at baseline (the first day on MV) and during the period of invasive respiratory support (maximum or minimum, depending on the parameter). Categorical variables are expressed as numbers (\%), and continuous variables as median (IQR). Ventilatory ratio is defined as [minute ventilation ( $\mathrm{ml} / \mathrm{min}) \times \mathrm{PaCO}_{2}$ $(\mathrm{mmHg}) /$ (predicted body weight $\times 100 \times 37.5)]$

ARDS acute respiratory distress syndrome, $\mathrm{PEEP}$ positive end-expiratory pressure, $\mathrm{PaO}_{2} / \mathrm{FiO}_{2}$ partial pressure of arterial oxygen to inspiratory oxygen fraction ratio, $\mathrm{PaCO}_{2}$ partial pressure of carbon dioxide, $E C M O$ extracorporeal membrane oxygenation, ICU intensive care unit

$* 0.05$ after Benjamini-Hochberg correction

\# Mean $\mathrm{FiO}_{2}$ was calculated with the values reported during the overall period under invasive mechanical ventilation 
$p=0.026$ ] and moderate ARDS [hazard ratio (RR) 0.69 (95\% CI 0.47-0.97), $p=0.035$ ] compared to severe ARDS (Fig. 3). Sensitivity analysis for outcomes are shown in Figure S8. The ICU discharge and risk of 28-day mortality was not affected by Crs (Table S3 and Figure S9). The association of driving pressure and Pplat on outcomes is shown in Figure S10. Patients classified as moderate ARDS who, after $24 \mathrm{~h}$ of MV moved to mild ARDS, had a strong trend towards a lower 28 day mortality, than those who remained classified as moderate ARDS on day 2, but this association was not statistically significant [HR: 0.55 (95\% CI 0.26-1.15), $p$ value $=0.113$ ]. In general, being treated in specific hospitals had no impact on outcomes (Figure S11).

\section{Discussion}

In this multicenter, observational study in 742 mechanically ventilated patients with COVID-19 ARDS, predominantly older, male patients with comorbid conditions, with a median ICU length of stay of 21 days, the majority had moderate ARDS, and greater than $80 \%$ had low Crs. The values of Crs, Pplat and driving pressure were very similar to previously published cohorts of ARDS patients. On average, patients were managed with low VT and moderate PEEP levels within the standard paradigm of lung-protective VT. Adjunctive therapies, such as RMs or prone position, were used frequently. Mortality at 28-days was similar to patients with non-COVID ARDS.

As previously reported for patients with COVID-19, the most common comorbidities were arterial hypertension and obesity $[4,18]$. The main reason for ICU admission in our study was acute respiratory failure, although the SOFA scores indicated more than one organ dysfunction. Hemodynamic impairment requiring vasopressors was the most common associated organ dysfunction, in agreement with the findings of Goyal et al. [18], where $95 \%$ of their invasively ventilated patients required vasopressors. Of note, the median time from symptom onset to hospital admission was similar to that reported previously [19]. On average, hypoxia was severe within the range of previous reports on COVID-19 and nonCOVID-19 ARDS patients [4, 13, 20]. The proportions of severe COVID-19 ARDS patients were greater than those reported in epidemiological studies of non-COVID-19 ARDS [14] (Table S6). However, we found, as previously reported, a marked redistribution of ARDS severity $24 \mathrm{~h}$ after ARDS diagnosis [21]. This reduction in the percentage of patients with severe ARDS criteria may be related to positive pressure ventilation by itself, to the effectiveness of adjunctive measures, or (unlikely) the natural history of the disease process (Fig. 2). Although it was not the aim of this analysis, it is important to highlight that some investigators argue that the degree of ARDS severity is best evaluated $24 \mathrm{~h}$ after assessing $\mathrm{PaO}_{2} / \mathrm{FiO}_{2}$ under certain ventilatory settings [22].

Our findings in a cohort of over 700 patients are in line with preliminary studies of COVID-19 ARDS patients $[23,24]$. We found no significant differences when baseline Crs, Pplat and driving pressure were compared to non-COVID-19 ARDS observational and randomized ARDS studies (Table S6). These comparisons were not based on a formal meta-analysis, and thus, these comparisons serve to demonstrate that there are no major differences in these baseline values for COVID-19 ARDS compared to non-COVID-19 ARDS.

In general, compliance with lung-protective ventilation was high, independent of the degree of severity of the disease process and somewhat higher on average than in previous observational studies of non-COVID-19 ARDS patients $[13,20]$. This finding was likely due to a greater awareness that these patients had ARDS. As reported in the LUNG SAFE study, one of the main problems in not complying with lung protection strategies was the underdiagnosis of ARDS [25]. In our cohort, invasive MV was maintained within the limits of lung-protective ventilation, as defined using a VT $\leq 8 \mathrm{ml} / \mathrm{kg} \mathrm{PBW}$, Pplat $<30$ $\mathrm{cmH}_{2} \mathrm{O}$, and a driving pressure $\leq 15 \mathrm{cmH}_{2} \mathrm{O}$ [26]. In our cohort, RMs were the most frequent adjunctive therapies used, followed by prone position, and NMBA. These findings are in contrast to reported practice in nonCOVID-19 severe ARDS patients [4, 13, 20]. Surprisingly, the use of RMs was not influenced by ARDS severity or by Crs. Both RMs and prone ventilation are usually performed to improve arterial oxygenation, and reduce ventilator-induced lung injury $[27,28]$. The impact of these maneuvers depends on the recruitability of the lung, which has been shown to be variable in COVID-19 ARDS [29].

In our experience, respiratory drive in COVID-19 ARDS patients appeared to be high, despite adequate sedation, making it difficult to maintain low transpulmonary pressures, which could lead to self-inflicted lung

(See figure on next page.)

Fig. 3 Time to event curves using Kaplan-Meier with univariable Cox regression. The probability of discontinuation from mechanical ventilation and the probability of ICU discharge increase with decreasing ARDS. The 28-day probability of death was higher in severe ARDS. ICU intensive care unit, ARDS acute respiratory distress syndrome 
1- Probability of discontinuing mechanical ventilation

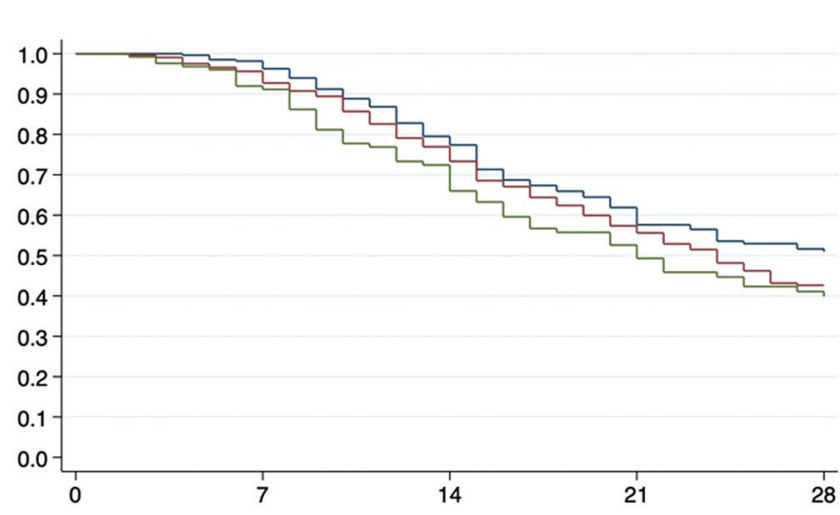

Log-Rank $\mathrm{p}=0.063$

Cl) moderate vs severe: $1.22(0.95-1.56) ; p=0.114$

Hazard Ratio $(95 \% \mathrm{Cl})$ mild vs severe: $1.42(1.04-1.92) ; p=0.026$

\section{1- Probability of ICU discharge}

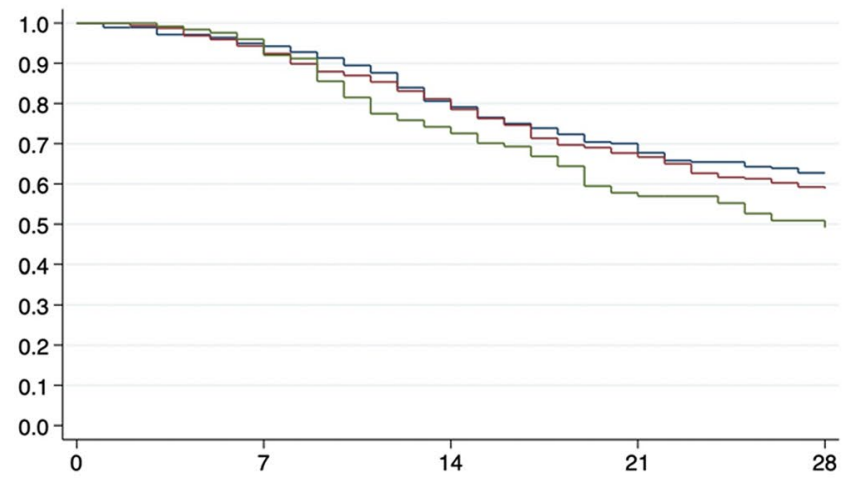

Log-Rank $p=0.044$

(1) moderate vs severe: $1.12(0.87-1.46) ; \mathrm{p}=0.380$

Hazard Ratio $(95 \% \mathrm{Cl})$ mild vs severe: $1.49(1.08-2.04) ; p=0.014$

Probability of 28-day survival

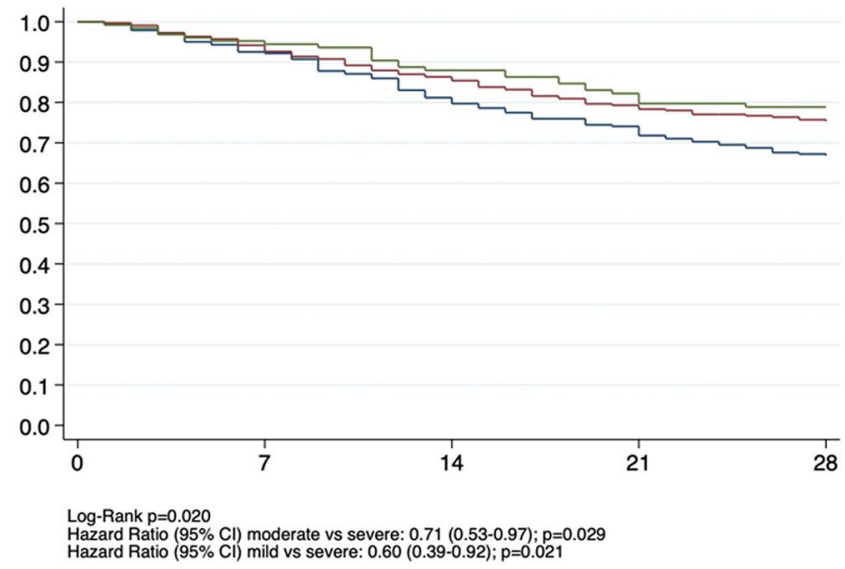


injury [30]. This bedside observation may explain the large number of patients in whom NMBA were used. Another reason for the high use of NMBA could be the large number of patients treated in the prone position; although NMBA are not required, they are often used in these patients, as reported in previous studies [17]. Nonetheless, the protective effects of NMBA have been seriously questioned in ARDS $[16,31]$. The probability of being discharged from the ICU was influenced by ARDS severity but not by Crs, as reported in studies of nonCOVID-19 ARDS patients [13]. All-cause 28-day mortality was similar or lower than previously published for non-COVID (Table S6) and COVID-19 ARDS [4, 32, 33] patients.

This study has several strengths. The study was very large with over 700 patients from 36 ICUs. As well, this is the first study to provide very detailed physiological data and ventilation strategies during the entire ventilatory period in COVID-19 ARDS patients. However, we acknowledge a number of limitations. First, our study design did not allow us to analyze potential associations of ventilatory strategies with outcomes. Second, we were unable to determine why certain therapeutic approaches were used; for example, how PEEP was adjusted (pragmatic or individualized approach), or why adjunctive therapies (RM, prone position) were applied (usual practice, refractory hypoxemia, etc.), or the indications and timings of ECMO, or corticosteroids. Third, Cox regression analysis was not adjusted for confounders. The main reasons were the low grade of imbalances in the groups in the relevant baseline variables. Fourth, due to the critical moment of the pandemic, and that most participating centers had rapidly reached ICU saturation and intensivists were forced to make difficult decisions, we did not collect the total number of patients admitted to participant ICUs during the study period. Finally, it is plausible that due to the burden of care experienced by participating clinicians during the study period, the ventilatory strategy and specifically, the use of adjunctive therapies may not be representative of clinical practice in non-pandemic circumstances.

In conclusion, in this large series, COVID-19 ARDS patients appear to have similar physiological features (including respiratory system compliance, plateau pressure and driving pressure) to other causes of ARDS. Compliance with lung-protective ventilation was high, and the risk of 28-day mortality increased with the severity of ARDS, but was not greater than other studies in non-COVID-19 ARDS patients.

\section{Electronic supplementary material}

The online version of this article (https://doi.org/10.1007/s00134-020-06192-2) contains supplementary material, which is available to authorized users.

\section{Author details}

${ }^{1}$ Department of Anesthesiology and Critical Care, Institut D'investigació August Pi I Sunyer, Hospital Clínic, Villarroel 170, 08025 Barcelona, Spain. ${ }^{2}$ CIBER de Enfermedades Respiratorias, Instituto de Salud Carlos III, Madrid, Spain. ${ }^{3}$ Department of Surgical Sciences, Hedenstierna Laboratory, Uppsala University Hospital, Uppsala, Sweden. ${ }^{4}$ Intensive Care Unit, Hospital Universitario La Princesa, Madrid, Spain. ${ }^{5}$ Department of Anesthesiology and Critical Care, Hospital de Cruces, Barakaldo, Vizcaya, Spain. ${ }^{6}$ Department of Preventive Medicine and Public Health, Medical School, University of Navarra, Pamplona, Spain. ${ }^{7}$ Ubikare Technology, Vizcaya, Spain. ${ }^{8}$ Department of Anesthesiology and Critical Care, Hospital Universitario Río Hortega, Valladolid, Spain. ${ }^{9}$ Department of Nutrition, Harvard TH Chan School of Public Health, Boston, USA. ${ }^{10}$ CIBER de Fisiopatología de La Obesidad.Y Nutrición, Instituto de Salud Carlos III, Madrid, Spain. ${ }^{11}$ Li Ka Shing Knowledge Institute, St Michael's Hospital, Toronto, ON, Canada. ${ }^{12}$ Department of Medicine, University of Toronto, Toronto, ON, Canada. ${ }^{13}$ Multidisciplinary Organ Dysfunction Evaluation Research Network, Research Unit, Hospital Universitario Dr. Negrín, Las Palmas de Gran Canaria, Spain.

\section{Acknowledgements}

The members for the COVID-19 Spanish ICU Network are listed as Hospital Clínic de Barcelona, Department of Anesthesiology and Critical Care: Carlos Ferrando, Graciela, Martínez-Pallí, Jordi Mercadal, Guido Muñoz, Ricard Mellado, Adriana Jacas, Marina Vendrell, Gerard Sánchez-Etayo, Amalia Alcón, Isabel Belda, Mercé Agustí, Albert Carramiñana, Isabel Gracia, Miriam Panzeri, Irene León, Jaume Balust, Ricard Navarro, María José Arguís, Maria José Carretero, Cristina Ibáñez, Juan Perdomo, Antonio López, Manuel López, Tomás Cuñat, Marta Ubré, Antonio Ojeda, Andrea Calvo, Eva Rivas, Paola Hurtado, Roger Pujol, Nuria Martín, Javier Tercero, Pepe Sanahuja, Marta Magaldi, Miquel Coca, Elena del Rio, Julia Martínez-Ocon, Paula Masgoret, Angel Caballero, Raquel Risco, Lidia Gómez, Nicolás de Riva, Ana Ruiz, Beatriz, Tena, Sebastián Jaramillo, Jose María Balibrea, Francisco Borja de Lacy, Ana Otero, Ainitze Ibarzabal, Raquel Bravo, Anna Carreras, Daniel Martín-Barreda, Alfonso Jesús Alias, Mariano Balaguer, Jorge Aliaga, Alex Almuedo, Joan Ramón Alonso, Rut Andrea, Gerard Sergi Angelès, Marilyn Arias, Fátima Aziz, Joan Ramon Badía, Enric Barbeta, Toni Torres, Guillem Batiste, Pau Benet, Xavi Borrat, María Borrell, Ernest Bragulat, Inmaculada Carmona, Manuel Castellà, Pedro Castro, Joan Ceravalls, Oscar Comino, Claudia Cucciniello, Clàudia De Deray, Oriol De Diego, Paula De la Matta, Marta Farrero, Javier Fernández, Sara Fernández, Anna Fernández, Miquel Ferrer, Ana Fervienza, María Tallo Forga, Daniel Forné, Clàudia Galán, Andrea Gomez, Eduard Guasch, Maria HernándezTejero, Beltrán Jiménez, Pere Leyes, Teresa López, José Antonio Martínez, Jose Muñoz, Ricard Navarro, Josep Maria Nicolás, José Tomás Ortiz, Anna Peiró, Manuel Pérez, Esteban Poch, Margarida Pujol, Eduard Quintana, Bartomeu Ramis, Enric Reverter, Irene Rovira, Pablo Ruiz, Elena Sandoval, Stefan Schneider, Oriol Sibila, Carla Solé, Alex Soriano, Dolors Soy, M. Suárez, Adrián Téllez, Néstor David Toapanta, Antoni Torres, Xavier Urra. Hospital Universitario Río Hortega: César Aldecoa, Alicia Bordell, Silvia Martín, Judith Andrés. Hospital Universitario Cruces: Alberto Martínez Ruiz, Gonzalo Tamayo Medel, Iñaki Bilbao Villasante, Fernando Iturri Clavero, Covadonga Peralta Álvarez, Julia T. Herrera, Andrea García Trancho, Iñaki Sainz Mandiola, Carmen Ruano Suarez, Angela Ruiz Bocos, Eneritz Urrutia Izagirre, Pablo Ortiz de Urbina Fernández, Naiara Apodaka, Leire Prieto Molano, Eunate Ganuza, Iratxe Vallinas, Karmele de Orte, Celia González Paniagua, Gemma Ortiz, Mireia Pérez, Marta López, Estíbaliz Bárcena, Erik Urutxurtu, Maria Jesús Maroño, Blanca Escontrela. Aitziber Ereñozaga, Deiene Lasuen, Ainhoa Zabal, Ane Guereca. Hospital del Mar: Ramón Adalia, Luigi Zattera, Irina Adalid Hernandez, Leire Larrañaga Altuna, Aina Serrallonga Castells, Adriana Vilchez Garcia, María Núñez, Lorena Román. Hospital General Universitario De Ciudad Real: Francisco Javier Redondo Calvo, Ruben Villazala González, Victor Baladron González, Patricia Faba, Omar Montenegro, Natalia Bejarano Ramírez. Complejo Asistencial Universitario León: Sergio Marcos Contreras, Alejandro Garcia Rodríguez, Saleta Rey Vázquez, Cristina Garcia Pérez, Eva Higuera Miguelez, Irene Pérez Blanco, David García Rivera. Hospital Urduliz: Ane Martín de la Fuente, Marta Pardo, Vanessa Rodriguez, Unai Bengoetxea. Hospital Universitario de la Princesa: Fernando Ramasco, Sheila Olga Santidrián Bernal, Alvar Santa Cruz Hernando. Hospital Povisa: Beatriz Domínguez, Ana Vázquez Lima. Hospital Ramón y Cajal: Ángel Candela, Ismael A Acevedo Bambaren, Maria Isabel Albala Blanco, Paloma Alonso Montoiro, Fernando Álvarez Utrera, Juan Avellanosa Esteruelas, Amal Azzam López, Alberto José Balvis Balvis, María Beltrán Martín, Jacobo Benatar Haserfaty, Alberto Berruezo Camacho, Laura 
Betolaza Weimer, Maria del Mar Carbonell Soto, Cristina Carrasco Seral, Cristina Cerro Zaballos, Elizabeth Claros Llamas, Pilar Coleta Orduna, Ingrid P. Cortes Forero, Pascual Agustín, Crespo Aliseda, María Angélica de Pablo Pajares, Yolanda Díez Remesal, Trinidad Dorado Díaz, Noemí Echevarría Blasco, María Elena Elías Martín, Javier Felices Triviño, Natalia Fernández López, Cristina Fernández Martín, Natalia Ferreiro Pozuelo, Luis Gajate Martín, Clara Gallego Santos, Diego Gil Mayo, María Gómez Rojo, Claudia González Cibrián, Elena Herrera López, Borja Hinojal Olmedillo, Berta Iglesias Gallego, Sassan Khonsari, María Nuria Mane Ruiz, María Manzanero Arroyo, Ana María Mariscal Ortega, Sara Martín Burcio, María del Carmen Martín González, Ascensión Martín Grande, Jose Juan Martín López, Cecilia Martín Rabes, Marcos Martínez Borja, Nilda Martínez Castro, Adolfo Martínez Pérez, Snejana Matcan, Cristina Medrano Viñas, Lisset Miguel Herrera, Adrián Mira Betancur, María Montiel Carbajo, Javier Moya Moradas, Lorena Muñoz Pérez, Mónica Nuñez Murias, Eva Ordiales González, Óscar Ordoñez Recio, Miguel Ángel Palomero Rodriguez, Diego Parise Roux, Lucia Pereira Torres, David Pestaña Lagunas, Juana María Pinto Corraliza, Marian Prieto Rodrigo, Inmaculada Rodriguez Diaz-Regaño, David Rodriguez Esteban, Víctor Rojas Pernia, Álvaro Ruigómez Saiz, Bárbara Saavedra Villarino, Noemí Samaranch Palero, Gloria Santos Pérez, Jaume Serna Pérez, Ana Belén Serrano Romero, Jesús Tercero López, Carlos Tiscar García, Marta de la Torre Concostrina, Eva María Ureta Mesa, Eva Velasco Olarte, Judith Villahoz Martínez, Raúl Villalaba Palacios, Gema Villanueva García, Cristina Vogel de Medeiros. Hospital Universitario Severo Ochoa: Soraya Gholamian Ovejero, Marta Vicente Orgaz, Patricia Lloreda Herradon, Cristina Crespo Gómez. Hospital Universitario de Gran Canaria Dr. Negrín: Tatiana Sarmiento-Trujillo. Hospital de Terrassa: Noemí García Medina, María Martínez García, Carles Espinós Ramírez, Nabil Mouhaffel Rivero, Jose Antonio Bernia Gil. Hospital Central de la Cruz Roja San José y Santa Adela: Sonsoles Martín. Hospital de la Santa Creu i Sant Pau: María Victoria Moral, Josefina Galán, Pilar Paniagua, Sergio Pérez, Albert Bainac, Ana Arias, Elsa Ramil, Jorge Escudero. Clínica Universidad de Navarra: Pablo Monedero, Carmen Cara, Andrea Lara, E. Mendez Martínez, Jorge Mendoza, Iñigo Rubio Baines. Medicina Preventiva y Salud Pública: Alfredo Gea, Alejandro Montero. Hospital Universitario Dr. Peset Aleixandre: Rocío Armero Ibañez, Juan Vicente Llau Pitarch, Fernando Rauer Alcover, Cristina Álvarez Herreros, Cyntia Sánchez Martín, Lucía López Ocáriz Olmos, Marta Navas Moruno. Complejo Hospitalario Universitario de Cáceres: Fernando García Montoto. MF. Mirón Rodriguez, Laura Fuentes Coco, Cristina Hernández Gamito, Antonio Barba Orejudo, Luis Gerardo Smith Vielma, Yasmina González Marín, Francisco de Borja Amador Penco, Marta Donoso Domínguez, Silvia Esquivel Ramírez. Hospital Clínico Universitario de Valencia: José Antonio Carbonell, Berta Monleón López, Sara Martínez-Castro, Gerardo Aguilar. Hospital Universitario a Coruña: María Gestal, Pablo Casas, Angel Outeiro Rosato, Andrea Naveiro Pan, María Alonso Portela, Adrián García Romar, Eva Mosquera Rodríguez, Diego Ruanova Seijo, Pablo Rama Maceiras. Complexo Universitario de Ferrol: Francisco Castro-Ceoane, Esther Moreno López. Hospital Clínico Universitario Lozano Blesa: Sergio Gil, Julia Guillén Antón, Patricia García-Consuegra Tirado, Aurora Callau Calvo, Laura Forés Lisbona, María Carbonell Romero, Belén Albericio Gil, Laura Pradal Jarne, María Soria Lozano, Diego Loscos López, Andrea Patiño Abarca. Universal Doctors: Jordi Serrano. UBIKARE: Javier Pérez-Asenjo, Ángel Díez-Domínguez, Ion Zubizarreta, Jon Ramos, losu Fernández. Hospital Universitario La Paz: Emilio Maseda, Alejandro Suárez de la Rica, Javier Veganzones, Itziar Insausti, Javier Sagra, Sofía Díaz Carrasco, Ana Montero Feijoo, Julio Yagüe. Hospital Universitario Gregorio Marañon: Ignacio Garutti. Hospital San Joan Despí Moises Broggi: Eva Bassas Parga, Carmen Deiros Garcia, Elisenda Pujol Rosa, Ana Tejedor Navarro, Roser Font Gabernet, Maria José Bernat, Meritxell Serra Valls, Cristina Cobaleda Garcia-Bernalt, Jesus Fernanz Anton, Adriana Aponte Sierra, Lucia Gil Gomez, Olaia Guenaga Vaqueiro, Susana Hernandez Marin, Laura Pardo Pinzon, Sira Garcia Aranda, Carlos Briones Orejuela, Edgar Cortes Sanchez, Alejandro Romero Fernandez, Esther Fernández Sanjosé, Patricia I glesias Garsabal, Guillermo Isidro Lopez, Ana Vicol, Sara Espejo Malagon, Maria Sanabra Loewe, Laura Grau Torradeflo, Lourdes Blanco Alcaide, Gloria Buenaventura Sanclemente, Pere Serra Pujol, Gustavo Cuadros Mendoza, Miroslawa Konarska, Fedra Bachs Almenara, Agnieszka Golska, Aleix Carmona Blesa, Arantxa Mas Serra. Hospital Universitario Infanta Leonor: Javier Ripollés Melchor, Ana Nieto Moreno, Káteri Chao Novo, Sandra Gadín López, Elena Nieto Moreno, Bérénice Gutiérrez Tonal, Elena Lucena de Pablo, Barbara Algar Yañez, Beatriz Vázquez Rivero, Beatriz Nozal Mateo, Marina de Retes, Norma Aracil Escoda, Cristina Gallardo Mayo, Rosa Sanz González, Alicia Ruiz Escobar, MariaLaura Pelegrina López, Marina Valenzuela Peña, David Stolle Dueñas, Ane Abad Motos, Alfredo Abad-Gurumeta, Ana Tirado Errazquin,
Elena Sáez Ruiz, Nerea Gómez Perez, Francisco de Borja Bau González. Hospital sanitas CIMA: Cesar Morcillo Serra, Jessica Souto Higueras. Hospital Universitario y Politécnico La Fé: Rosario Vicente, Raquel Ferrandis, Silvia Polo Martín, Azucena Pajares Moncho, Ignacio Moreno Puigdollers, Juan Pérez Artacho Cortés, Ana Moret Calvo, Ana Pi Peña, María Catalán Fernández. Complexo hospitalario Universitario de Pontevedra: Marina Varela, Pilar Diaz. Hospital Arnau de Vilanova: María Isabel Forés Chiva. Hospital General de Alicante: A. Javier Agulló. Hospital Universitario Infanta Sofía: Antonio Pérez, María Barrionuevo, Paloma Medrano, Cristina Sanz, Manuel Ortega. Hospital Universitario San Juan de Alicante: Maria Galiana. Hospital Nuestra Señora de Meritxell SAAS: Antoni Margarit, Válerie Mourre del Rio, Eva Heras Muxella, Anna Vidal.

\section{Author contributions}

All authors contributed to the study conception and design. Material preparation, data collection and analysis were performed by CF, RM, MM, AG, EA, CA and GM-P. The first draft of the manuscript was written by CF and all authors commented on previous versions of the manuscript. All authors read and approved the final manuscript.

\section{Funding}

Instituto de Salud Carlos III, Madrid, Spain (\#CB06/06/1088; \#PI16/00049; \#PI18/01611; \#PI19/00141); and Canadian Institutes of Health Research (CIHR) FDN143285, and OV3-170344.

\section{Data availability}

\section{By request to the corresponding author.}

\section{Code availability}

\section{Not applicable.}

\section{Compliance with ethical standards}

\section{Conflicts of interest}

The authors declare no conflicts of interest in relation to this manuscript.

\section{Ethics approval}

The study was approved by the referral Ethics Committee of Hospital Clínic, Barcelona, Spain (code number HBC/2020/0399).

\section{Consent to participate}

This is an observational study. The need for written informed consent from participants was considered by each participating center.

\section{Consent for publication}

Not applicable.

\section{Publisher's Note}

Springer Nature remains neutral with regard to jurisdictional claims in published maps and institutional affiliations.

Received: 12 May 2020 Accepted: 17 July 2020

Published online: 29 July 2020

\section{References}

1. Wenjie T, Xiang Z, Xuejun M, et al. (2020) A novel coronavirus genome identified in a cluster of pneumonia cases - Wuhan, China 2019-2020. https://weekly.chinacdc.cn/en/article/id/a3907201-f64f-4154-a19e4253b453d10c. Accessed 03 July 2020

2. Grasselli G, Pesenti A, Cecconi M (2020) Critical care utilization for the COVID-19 outbreak in Lombardy, Italy: early experience and forecast during an emergency response. JAMA. https://doi.org/10.1001/ jama.2020.4031

3. Ruan Q, Yang K, Wang W et al (2020) Clinical predictors of mortality due to COVID-19 based on an analysis of data of 150 patients from Wuhan. China Intensive Care Med 46:846-848. https://doi.org/10.1007/s0013 4-020-05991-x 
4. The COVID-19 Lombardy ICU Network (2020) Baseline characteristics and outcomes of 1591 patients infected with SARS-CoV-2 admitted to ICUs of the Lombardy region, Italy. JAMA 323:1574-1581. https://doi. org/10.1001/jama.2020.5394

5. Huang C, Yeming W, Xingwang L et al (2020) Clinical features of patients infected with 2019 novel coronavirus in Wuhan, China. Lancet 395:497506. https://doi.org/10.1016/S0140-6736(20)30183-5

6. Gattinoni L, Coppola S, Cressoni M (2020) Covid-19 does not lead to a "typical" acute respiratory distress syndrome. Am J Respir Crit Care Med 201:1299-1300. https://doi.org/10.1164/rccm.202003-0817LE

7. Gattinoni L, Chiumello E, Caironi P et al (2020) COVID-19 pneumonia: different respiratory treatment for different phenotypes? Intensive Care Med 46:1099-1102. https://doi.org/10.1007/s00134-020-06033-2

8. Marini JJ, Gattinoni L (2020) Management of COVID-19 respiratory distress. JAMA. https://doi.org/10.1001/jama.2020.6825

9. Ranieri VM, Rubenfeld GD, Thompson BT, ARDS Definition Task Force et al (2012) Acute respiratory distress syndrome: the Berlin Definition. JAMA 307:2526-2533. https://doi.org/10.1001/jama.2012.5669

10. Von Elm E, Altman DG, Egger M, Pocock SJ, Gotzsche PC, Vanderbrouche JP, STROBE Initiative (2007) Strengthening the reporting of observational studies in epidemiology (STROBE) statement: guidelines for reporting observational studies. BMJ 335:806-808. https://doi.org/10.1136/ bmj.39335.541782.AD

11. WHO (2020) Clinical management of severe acute respiratory infection when novel coronavirus ( $\mathrm{nCoV}$ ) infection is suspected: interim guidance, 25 January 2020. Published January 25. https://apps.who.int/iris/handl e/10665/330854. Accessed 15 Apr 2020.

12. Brock GN, Barnes C, Ramirez JA, Myers J (2011) How to handle mortality when investigating length of hospital stay and time to clinical stability. BMC Med Res Methodol 11:144. https://doi. org/10.1186/1471-2288-11-144

13. Bellani G, Laffey JG, Pham T et al (2016) Epidemiology, patterns of care, and mortality for patients with acute respiratory distress syndrome in intensive care units in 50 countries. JAMA 315:788-800. https://doi. org/10.1001/jama.2016.0291

14. Kacmarek RM, Villar J, Sulemanji D, Open Lung Approach Network et al (2016) Open lung approach for the acute respiratory distress syndrome: a pilot, randomized controlled trial. Crit Care Med 44:32-42. https://doi. org/10.1097/CCM.0000000000001383

15. Writing Group for the Alveolar Recruitment for Acute Respiratory Distress Syndrome Trial (ART) Investigators, Cavalcanti AB, Suzumura ÉA et al (2017) Effect of lung recruitment and titrated positive end-expiratory pressure (PEEP) vs low PEEP on mortality in patients with acute respiratory distress syndrome: a randomized clinical trial. JAMA 318:1335-1345. https://doi.org/10.1001/jama.2017.14171

16. Moss M, Ulysse CA, Angus DC, National Heart, lung, and blood institute PETAL clinical trials network (2019) Early neuromuscular blockade in the acute respiratory distress syndrome. N Engl J Med 380:1997-2008. https ://doi.org/10.1056/NEJMc1908874

17. Guérin C, Reignier J, Richard JC et al (2013) Prone positioning in severe acute respiratory distress syndrome. N Engl J Med 368:2159-2168. https ://doi.org/10.1056/NEJMoa1214103

18. Goyal P, Choi JJ, Pinheiro LC et al (2020) Clinical characteristics of COVID-19 in New York city. N Engl J Med 382:2372-2374. https://doi. org/10.1056/NEJMc2010419

19. Yang X, Yu Y, Xu J et al (2020) Clinical course and outcomes of critically ill patients with SARS-CoV-2 pneumonia in Wuhan, China. Lancet Respir
Med S2213-2600(20):30079-30085. https://doi.org/10.1016/S2213 $-2600(20) 30079-5$

20. Villar J, Blanco J, Añón JM et al (2011) The ALIEN study: incidence and outcome of acute respiratory distress syndrome in the era of lung protective ventilation. Intensive Care Med 37:1932-1941. https://doi.org/10.1007/ s00134-011-2380-4

21. Madoto F, Pham T, Bellani G et al (2018) Resolved versus confirmed ARDS after 24h: insights from the LUNG SAFE study. Intensive Care Med 44:564-577. https://doi.org/10.1007/s00134-018-5152-6

22. Villar J, Fernández RL, Ambrós A et al (2015) A clinical classification on the acute respiratory distress syndrome for predicting outcome and guiding medical therapy. Crit Care Med 43:346-353. https://doi.org/10.1097/ CCM.0000000000000703

23. Ziehr DR, Alladina J, Petri CR et al (2020) Respiratory pathophysiology of mechanically ventilated patients with COVID-19: a cohort study. Am J Respir Crit Care Med 201:1506-1564. https://doi.org/10.1164/rccm.20200 4-1163LE

24. Schenck EJ, Hoffman K, Goyal P et al (2020) Respiratory mechanics and gas exchange in COVID-19 associated respiratory failure. Ann Am Thorac Soc. https://doi.org/10.1513/AnnalsATS.202005-427RL

25. Bellani G, Pham T, Laffey J (2020) Missed or delayed diagnosis of ARDS: a common and serious problem. Intensive Care Med 46:1180-1183. https ://doi.org/10.1007/s00134-020-06035-0

26. Pistillo N, Fariña O (2018) Driving airway and transpulmonary pressure are correlated to VILI determinants during controlled ventilation. Intensive Care Med 44:674-675. https://doi.org/10.1007/s00134-018-5092-1

27. Pensier J, de Jong A, Hajjej Z et al (2019) Effect of lung recruitment maneuver on oxygenation, physiological parameters and mortality in acute respiratory distress syndrome patients: a systematic review and meta-analysis. Intensive Care Med 45:1691-1702. https://doi.org/10.1007/ s00134-019-05821-9

28. Guérin C, Beuret P, Constantin JM, Investigators of the APRONET Study Group, the REVA Network, the Réseau recherche de la Société Française d'Anesthésie-Réanimation (SFAR-recherche), and the ESICM Trials Group et al (2018) A prospective international observational prevalence study on prone positioning of ARDS patients: the APRONET (ARDS Prone Position Network) study. Intensive Care Med 44:22-37. https://doi. org/10.1007/s00134-017-4996-5

29. Beloncle FM, Pavlovsky B, Desprez C et al (2020) Recruitability and effect of PEEP in SARS-Cov-2-associated acute respiratory distress syndrome. Ann Intensive Care 10:55. https://doi.org/10.1186/s13613-020-00675-7

30. Spinelli E, Mauri T, Beitler J et al (2020) Respiratory drive in the acute respiratory distress syndrome: pathophysiology, monitoring, and therapeutic interventions. Intensive Care Med 46:606-618. https://doi. org/10.1007/s00134-020-05942-6

31. Slutsky AS, Villar J (2019) Early paralytic agents for ARDS? Yes, no, sometimes. N Engl J Med 380:2061-2063. https://doi.org/10.1056/NEJMe19056 27

32. Huang C, Wang Y, Li X et al (2020) Clinical features of patients infected with 2019 novel coronavirus in Wuhan, China. Lancet 395:497-506. https //doi.org/10.1016/S0140-6736(20)30183-5

33. Arentz M, Yim E, Klaff L et al (2020) Characteristics and outcomes of 21 critically ill patients with COVID-19 in Washington State. JAMA 323:16121614. https://doi.org/10.1001/jama.2020.4326 